\title{
Prenatal Diagnosis and Fetal Outcomes of Cystic Hygroma: Experience of a Tertiary Hospital
}

\author{
Betul YAKISTIRAN', Doruk Cevdi KATLAN', Tuncay YUCE', Feride SOYLEMEZ ${ }^{1}$ \\ Ankara, Turkey
}

ABSTRACT

OBJECTIVE: Cystic hygromas of nuchal origin are reported to be associated with fetal aneuploidy and structural anomalies in $50-80 \%$ of the cases. We aimed to report our experience on cystic hygromas via 18 cases.

STUDY DESIGN: We conducted a retrospective study based on a review of medical records of patients who had fetal septated cystic hygroma, diagnosed by ultrasonography, in the Ankara University School of Medicine, Department of Obstetrics and Gynecology, between 2012 and 2016. All patients were provided with genetic counselling and prenatal invasive diagnostic procedures were applied to obtain fetal karyotype.

RESULTS: We observed 18 cases of fetal cystic hygroma among these patients $(0.39 \%)$. The abnormal karyotypes were: Turner syndrome (45X0) in $3(16.7 \%)$, trisomy 21 in $2(11.1 \%)$, trisomy 18 in $1(5.6 \%)$ and mosaic Turner syndrome in $1(5.6 \%)$ patient.

CONCLUSION: The presence of cystic hygroma carries a high risk for aneuploidy and major structural malformations. Invasive prenatal diagnostic procedures for fetal karyotype analyses and parental counselling about poor perinatal prognosis is mandatory.

Keywords: Chromosomal disorders, Cystic hygroma, Prenatal diagnosis

Gynecol Obstet Reprod Med 2019;25(1):4-6

\section{Introduction}

Cystic hygroma is a congenital malformation characterized by the presence of abnormal fluid collection at sites of lymphatic-venous collection within neck, mediastinum, abdomen, and axillary region (1). It is also defined as a subgroup of lymphangiomas with the cystic variety, and filled with protein-rich fluid (2). Cystic hygroma is classified as septated and non-septated. The overall incidence of cystic hygroma is ap-

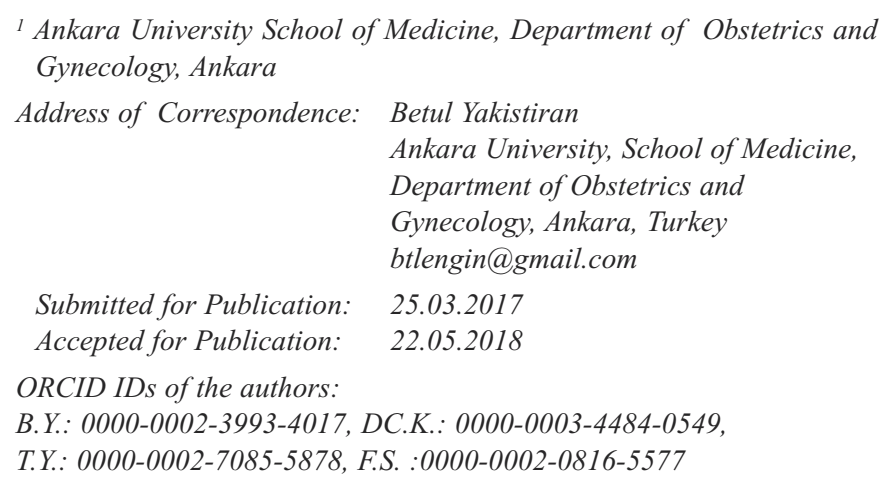

\begin{tabular}{|c|c|}
\hline \multirow{3}{*}{ 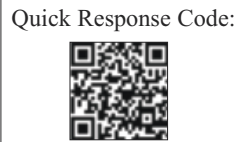 } & Access this article online \\
\hline & $\begin{array}{l}\text { Website: www.gorm.com.tr } \\
\text { e- mail: info@gorm.com.tr }\end{array}$ \\
\hline & DOI:10.201613/GORM.2018.789 \\
\hline
\end{tabular}

How to cite this article: Yakistiran B. Katlan DC. Yuce T. Soylemez F. Prenatal Diagnosis and Fetal Outcomes of Cystic Hygroma: Experience of a Tertiary Hospital. Gynecol Obstet Reprod Med 2019;25(1):4-6 proximately $1 / 1000-6000$ in births and $1 / 750$ in spontaneous abortions (3).

Cystic hygromas of nuchal origin are reported to be associated with fetal aneuploidy and structural anomalies in 50$80 \%$ of the cases (1). Although cystic hygromas diagnosed in the first trimester are associated with trisomy 21, 18, and 13, cases diagnosed in early second trimester seem to be commonly associated with monosomy X. Euploid fetuses with cystic hygroma may have major structural abnormalities, cardiac defects being the most encountered ones. Other coexisting non-cardiac defects may be hydrocephalus, skeletal defects, hypoplasia of the lung and developmental - genetic syndromes in decreasing order (4). Although the clinical significance of internal septations was not clearly documented, size of the lesions is an important prognostic factor for fetuses with cystic hygroma (5). In this study, we aimed to evaluate the gestational and fetal outcomes of fetuses with cystic hygroma.

\section{Material and Method}

We conducted a retrospective sectional study based on a review of medical records of patients who had fetal septated cystic hygroma, diagnosed by ultrasonography in the Ankara University School of Medicine, Department of Obstetrics and Gynecology, between December 2012 and February 2016. All scans were performed with using a Voluson E6 Expert TM 
(GE Healthcare, USA) multifrequency convex transducer at 2.0-7.0 MHz. Cystic hygroma was defined as an enlarged sonolucency with clearly visible septations extending along the fetal body axis, in contrast to NT, which was described as a nonseptated sonolucent area confined to the fetal neck (Figure 1) (6).

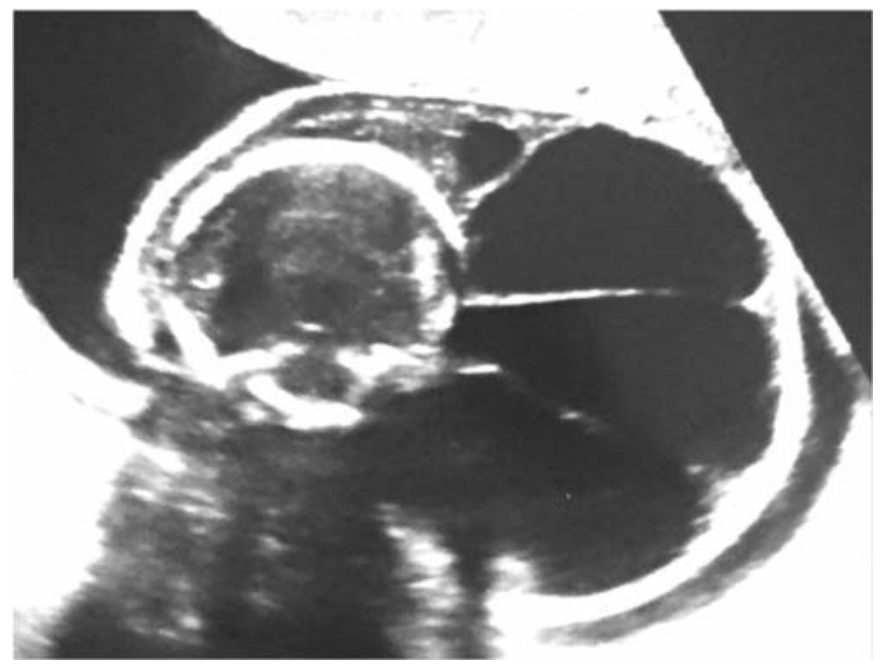

Figure 1: Cystic hygroma in transverse section of the head

Upon diagnosis of cystic hygroma, all patients were provided with genetic counselling and prenatal invasive diagnostic procedures were applied to obtain fetal karyotype. A complete fetal sonographic examination was then performed for the search of other structural anomalies in all patients with cystic hygroma. Depending on the gestational week at the time of diagnosis, either chronic villus sampling (CVS) or amniocentesis was the invasive diagnostic procedure. Data about karyotypes of fetuses, gestational and pediatric outcomes were collected either from the records of Obstetrics and Gynecology, Genetics and Pediatrics Departments of Ankara University or from direct phone calls to patients. This study was a retrospective sectional study; so ethical approval was not required by our institution.

\section{Results}

4589 singleton pregnancies were enrolled for first and sec- ond trimester sonography between December 2012 and February 2016. We observed 18 cases of fetal cystic hygroma among these patients $(0.39 \%)$. The details of the cases with cystic hygroma were summarized in table 1.

A normal karyotype was revealed in 10 cases $(55.5 \%)$, whereas 8 cases $(44.5 \%)$ had abnormal karyotypes. The abnormal karyotypes were: Turner syndrome $(45 \mathrm{X0})$ in $3(16.7 \%)$, trisomy 21 in $2(11.1 \%)$, trisomy 18 in $1(5.6 \%)$ and mosaic Turner syndrome in $1(5.6 \%)$ patient. Sonographic scan revealed associated findings in $8(44.5 \%)$ cases. These findings were hydrops and pleural effusion $(n=2,11.1 \%)$, hydrops $(n=1,5.6 \%)$, pes equinovarus $(n=1,5.6 \%)$, pectus carinatum $(\mathrm{n}=1,5.6 \%)$, perimembranous VSD $(\mathrm{n}=1,5.6 \%)$, multicystic dysplastic kidney $(n=1,5.6 \%)$ and short femur humerus length $(\mathrm{n}=1,5.6 \%)$. Termination of pregnancy was performed for 3 fetuses; one of them had trisomy 18 and the remaining two cases had trisomy 21 . There were two live births; the karyotypes were Turner syndrome and mosaic Turner. Other fetuses with Turner syndromes died. Cystic hygroma resolved during pregnancy in fetuses with normal karyotype.

\section{Discussion}

Cystic hygroma is a subgroup of lymphangioma and the main pathologic pathway is within the obstruction between the lymphatic and venous pathways leading to lymph accumulation. This condition can be diagnosed with sonography during fetal nuchal translucency measurement in the midsagittal plane in the first or early second trimester. The optimal time for assessment is approximately 10 to 14 weeks of gestation or when fetal crown-rump lengths (CRL) measurements are in between 45-84 mm. Detailed ultrasound examination and fetal chromosomal analyses are indicated due to high rates of fetal aneuploidy and coexisting structural malformations. Trisomies and monosomy $\mathrm{X}$ are commonly detected anomalies. At least half of the septated cystic hygroma cases were reported to be coexisting with chromosomal abnormalities. Recently, Scholl et al. reported that abnormal karyotype rates were $43.4 \%$ in fetuses with cystic hygroma detected when CRL measured below $45 \mathrm{~mm}$ and $73 \%$ fetuses with CRL was 45-84 mm (7). If nuchal translucency measurement was above

Table 1: Chromosomal characteristics and pregnancy outcomes of the patients with cystic hygroma

\begin{tabular}{lcc}
\hline Chromosomal abnormality & Number (\%) & Pregnancy outcome \\
\hline Overall & $7(38.9)$ & 1 Live birth \\
Turner syndrome & $3(16.7)$ & 1 Postpartum exitus \\
& & 1 IUFD \\
Trisomy 21 & $2(11.1)$ & 2 TOP \\
Trisomy 18 & $1(5.6)$ & 1 TOP \\
Mosaic Turner & $1(5.6)$ & 1 Live birth \\
\hline
\end{tabular}


$2 \mathrm{~mm}, 60 \%$ were related with abnormal karyotype and one fourth of these cases were detected to have trisomy 21 .

The diagnosis of cystic hygroma in the early second trimester worsens the prognosis. Bernstein et al., reported that both septation and size of cystic hygromas appeared to be the most important determinants of outcome (8). Cystic hygroma has a worse prognosis when associated with hydrops fetalis and cardiac, urologic, cerebral, skeletal malformations. We demonstrated several perinatal and postnatal bad outcomes such as spontaneous abortion, intrauterine fetal demise, and postpartum death. These associated anomalies further deteriorate the neonatal outcome of live-born fetuses (2).

Despite published data, the counseling of couples with fetal cystic hygroma after normal karyotype analysis and anomaly screening is quite challenging. When elective termination of pregnancy was chosen, couples refused termination for fetuses with multiple malformations. This situation gave rise to the limitations of our study. This study was about our clinical experience and we thought these kinds of reports could be helpful for the literature review.

In conclusion, the presence of cystic hygroma carries a high risk for aneuploidy and major structural malformations. Invasive prenatal diagnostic procedures for fetal karyotype analyses and parental counselling about poor perinatal prognosis is mandatory.

: Conflict of interest: The authors report no conflict of interest.

Funding: None

\section{References}

1. Podobnik M, Singer Z, Podobnik-Sarkanji S, Bulić M. First trimester diagnosis of cystic hygromata using trans- vaginal ultrasound and cytogenetic evaluation. J Perinat Med. 1995;23(4):283-91.

2. Gedikbasi A, Gul A, Sargin A, Ceylan Y. Cystic hygroma and lymphangioma: associated findings, perinatal outcome and prognostic factors in live-born infants. Arch Gynecol Obstet. 2007;276 (5):491-8. doi:10.1007/s00404007-0364-y

3. Chen CP, Liu FF, Jan SW, Lee CC, Town DD, Lan CC. Cytogenetic evaluation of cystic hygroma associated with hydrops fetalis, oligohydramnios or intrauterine fetal death: the roles of amniocentesis, postmortem chorionic villus sampling and cystic hygroma paracentesis. Acta Obstet Gynecol Scand. 1996;75(5):454-8.

4. Graesslin O, Derniaux E, Alanio E, Gaillard D, Vitry F, Quéreux C, et al. Characteristics and outcome of fetal cystic hygroma diagnosed in the first trimester. Acta Obstet Gynecol Scand. 2007;86(12):1442-6. doi:10.1080/00016 340701644843

5. Molina FS, Avgidou K, Kagan KO, Poggi S, Nicolaides KH. Cystic hygromas, nuchal edema, and nuchal translucency at 11-14 weeks of gestation. Obstet Gynecol. 2006;107(3):678-83. doi:10.1097/01.AOG.0000201979. 23031.32

6. Malone FD, Ball RH, Nyberg DA, Comstock CH, Saade GR, Berkoẃitz RL, et al. First-trimester septated cystic hygroma: prevalence, natural history, and pediatric outcome. Obstet Gynecol. 2005;106(2):288-94. doi: 10.1097/ 01.AOG.0000173318.54978.1f

7. Scholl J, Chasen ST. First trimester cystic hygroma: does early detection matter? Prenat Diagn. 2016;36(5):432-6. doi:10.1002/pd.4799

8. Bernstein HS, Filly RA, Goldberg JD, Golbus MS. Prognosis of fetuses with a cystic hygroma. Prenat Diagn. 1991;11(6):349-55. 